\title{
Some Thoughts about Health Informatics in Africa
}

\author{
Graham Wright
}

\author{
Department of Information Systems, Rhodes University, Grahamstown, South Africa
}

\section{Correspondence to:}

Professor Graham Wright, M.Phil, MBA, DN, Cert Ed, FBCS, CITP, MIASHI, SRN, RMN, Department of Information Systems, Rhodes University, Grahamstown, 6140, South Africa.

E-mail: prof.graham.wright@gmail.com
EJBI 2017; 14(1):75-76

Received: December 06, 2017

Accepted: December 06, 2017

published: January 08, 2018
I first started working in Africa 12 years ago and my initial impression was that there seemed to be lots of westernised "Capital" or "Large Cities" across the continent and the "rest" was Africa. There are a few real African large cities but the majority of Africa is urban or rural with a very different way of life compared to Europe or North America. Most of the populace has never used a computer and many have never seen one. Indeed in 2014 urban electrification was estimated at $45 \%$ of the population and only $19 \%$ had access in rural sub-Saharan Africa. Surely Hospitals use Electronic Health Systems? The answer is a firm yes that in the private sector, mainly insurance based, there are billing and payment systems based on ICD10 that record billable care. Indeed there exist systems in state hospitals, including PACS, largely in the developed cities [1]. The two most pervasive systems across the continent are DHIS and OpenClinic GA.

Murray, Betts [2] state that "The District Health Information System (DHIS), originally developed by the University of Oslo, and including the Health Information Systems Program (HISP) is a widely used public health information system, based in free and open source (FOSS) applications for collecting and sharing aggregated data from health facilities and transmitting it to higher levels in the public health system." DHIS is used in over 40 countries including South Africa, which in 2010 introduced a National System based on DHIS that uses registers exclusively as reporting tools, not as clinical tools. Registers are widely used in Public Health Monitoring and Evaluation, which has led to the development of data silos in the majority of countries using them which are of little use to clinicians. In Kenya all health programs are now integrated into DHIS2 for routine reporting.

OpenClinic GA is an open source integrated hospital information management system covering management of administrative, financial, clinical, laboratory, $\mathrm{x}$-ray, pharmacy, meals distribution and other data. Some of its features include a scheduling system, $3 \mathrm{BT}$ clinical thesaurus with validated coding aid for ICD-10 and ICPC-2, Snomed CT coding, laboratory order entry and results management, X-ray and pathology, pharmacy stock management and it integrates RxNorm based multi-lingual drug-drug interaction detection. It has installations in the following African countries: Burundi, Cameroon, Congo-Brazzaville, and Democratic Republic of the Congo, Gabon, Ivory Coast, Kenya, Mali, Nigeria, Senegal, Rwanda, Uganda, Sri Lanka, Tanzania and Zambia.

OpenClinic is a complete Hospital Information Management System (HIMS) that is Open Source, well supported and operational in some of the most troubled countries of Africa, some of which are HELINA and IMIA members. Marc Nyssen and Frank Verbeke, from Belgium, also help develop local solutions for Technical Support, HI Associations and Education and Training.

So what has all this got to do with Europe? DHIS2 is developed by HISP which is a global south-south-north collaborative network aiming at better health care in developing countries through the combination of research on and implementation of health information systems coordinated by the Department of Informatics at the University of Oslo (HISP UiO).

OpenClinic GA is developed by staff from BISI: Department of Biostatistics and Medical Informatics, Faculty of Medicine and Pharmacy, Vrije Universiteit Brussel, Brussels, Belgium and the Belgian Technical Cooperation, Brussels, Belgium and has a base in Burundi.

South Africa has, like other African countries, leap frogged the computer age with mobile phones; an estimated 92 million hand sets owned by a population of 62 million.

So why can we not use mobiles for Health? Access to the Internet is enjoyed by people in first world countries where internet access is cheap and readily available but not so in Africa where cost of data, power outages, education barriers, infrastructure and slow connection speeds are just some of the many challenges facing the mass roll out of internet on the continent. The Internet World Stats for Africa 2016 shows that only 9.3 per cent of people across the African continent are Internet users. Mobile phones ownership is growing in Africa regions in mobile phone use and Internet access, successfully sidestepping the era of desktop computers and landlines.

However many users only access free services for example there is a "call back" which is used to ask someone with airtime to call them, as they have no credit on their "pay as you go" phone. At locations that have free Wi-Fi students connect their mobile phones to use What's App for communication between themselves and Facebook and Instagram for social media [3]. The problem is that data is so expensive in Africa compared to other parts of the world. In addition the minimum wage in South Africa is 3000 rand, which is 178 Euro and the majority of students live on half of that amount. A $500 \mathrm{MB}$ data bundle costs 10 Euro or 150 Rand that will buy breakfast for 5 students! Technically Mobile Smart phones could be the answer for those privileged enough to be able to afford Internet access in Africa.

Globally it would, at first glance, appear that mobile phone health apps have a potential for supporting healthcare, especially in Africa, where mobile phones are more prevalent than desktops. However there are challenges that include non-integration with the 
health care delivery systems, lack of evidence of clinical effectiveness, and sustained user base. In December 2017 [4] a systematic review was published which looked to find scientific evidence on the efficacy of apps in promoting healthy lifestyles. It came to the conclusion that overall, the evidence so far showed a modest efficacy of apps in health promotion.

Unfortunately I know of no major success stories for the use of mobile phone based health related apps in Africa although there are claims. On examination the apps are either in development or can only be used to connect to specific programs running on a dedicated server.

\section{References}

[1] Wright G, O'Mahony D, Cilliers L. Electronic health information systems for public health care in South Africa: a review of current operational systems. Journal of Health Informatics in Africa. 2017; 4: 51-57.

[2]Murray PJ, Betts HJ, Wright G, Tshayingca-Mashiya NV. Health informatics education and capacity building in Eastern Cape Province, South Africa. Yearb Med Inform. 2009; 158-63.

[3]Wright G, Cilliers L, Niekerk EV, Seekoe E. The Next Stage of Development of Elearning at UFH in South Africa, In: 1th International Conference e-Learning. Lisbon, Portugal, 2017.

[4]Covolo L, Ceretti E, Moneda M, Castaldi S, Gelatti U. Does evidence support the use of mobile phone apps as a driver for promoting healthy lifestyles from a public health perspective? A systematic review of Randomized Control Trials. Patient Educ Couns. 2017; 100: 2231-2243. 